\title{
Mid-Epidemic Case Fatality Rate - The Case of COVID-19: Reducing the Impact of Wrong Risk Perception
}

\author{
Sukhpreet Deepon Patel ${ }^{1, *}$, Purnendu Nath ${ }^{2}$
}

\section{Sukhpreet Deepon Patel $^{1, *}$, Purnendu Nath ${ }^{2}$ 'Nowrosjee Wadia Maternity Hospital, Mumbai, Maharashtra, INDIA. \\ 'London Business School, Regent's Park, London, UK.}

\section{Correspondence Dr Sukhpreet Patel \\ Nowsrosjee Wadia Maternity Hospital, 91 Vallabh Apartments, 87 Bhulabhi Desai Road, Mumbai 400026, Maharashtra, INDIA. \\ Mobile no: +919321103838 \\ Email: sukhpreetpatel@gmail.com \\ History \\ - Submission Date: 18-05-2020 \\ - Revised Date: 16-07-2020 \\ - Accepted Date: 05-08-2020}

DOI : 10.5530/ijmedph.2020.3.26

Article Available online

http://www.ijmedph.org/v10/i3

\section{Copyright}

(C) 2020 Phcog.Net. This is an openaccess article distributed under the terms of the Creative Commons Attribution 4.0 International license.

\begin{abstract}
Background: In the midst of the current COVID-19 pandemic, the mortality risk being presented by both the media and some public health information providers is often misleading and so increasing the risk of suboptimal policy decisions and lower than desired voluntary compliance rates. Objectives: We make the following contributions. Methods: First and foremost, we explain why the obsessive focus on the post-epidemic retrospective Case Fatality Rate is misplaced and explain why the focus must necessarily be on a time-varying mid-epidemic measure. Second, we introduce a Mid-Epidemic Case Fatality Rate (ME-CFR) framework and explain, using this framework, why the mortality risk as often presented during the COVID 19 pandemic is inappropriate and downward biased. Third, we explain how the ME-CFR is related to the numbers being released for Cases, Deaths and Recovered. Fourth, we propose one such easy to use ME-CFR methodology for estimating the risk of death at a point in time when infected in the midst of an epidemic. Results: Present an illustrative example of such ME-CFR curves along with some commentary for 3 very different countries (India, South Korea, USA) for the current COVID 19 pandemic. Conclusion: We believe that if the professional publications and media switch to this measure of mid-epidemic outcomes, policymakers and the general public will make better decisions, leading to better outcomes for COVID 19 and for future epidemics and pandemics.

Key words: Mid-Epidemic CFR, COVID-19, Risk Perception.
\end{abstract}

\section{INTRODUCTION}

\section{A Curve will not Miss the Point}

In the midst of an epidemic or pandemic, in order to provide a quantitative measure to the public or policymakers the focus is typically towards an estimate of the 'probability of death' if infected by the relevant pathogen. It is important, of course, that, in retrospective studies, we understand what the postevent Case Fatality Rate (CFR) was for a given past epidemic. However, when mid-epidemic, a focus on that is about as useful as finding out which pier you will dock at when you finally reach shore, when you are currently bang in the middle of navigating your ship in the midst of a stormy ocean. So, it is important to appreciate that what matters is not the specific single point estimate in the distant future, but the time-varying curve that one is currently experiencing. In a nutshell, the focus ought to be on tackling a curve, not a point. We call this curve the Mid-Epidemic Case Fatality Rate (ME-CFR).

A further advantage of thinking about a curve is that of terminology within the story we tell ourselves. As presented eloquently by Brandt and Botelho ${ }^{1}$ for all people who are affected and can change the course of an epidemic, it is important to understand that using terminology that makes us passive recipients of what doom will ensue is harmful and likely to morph into a self-fulfilling prophecy. In that vein, we think of the ME-CFR as a trajectory whose course we have the tools, instruments and levers to alter. If all citizens have a feeling of being empowered by the terminology and language that we all use, we would collectively produce better ex post outcomes in an epidemic.

\section{MATERIALS AND METHODS}

\section{Data and Analysis}

Data used is from the repository for the 2019 Novel Coronavirus Visual Dashboard operated by the Johns Hopkins University Center for Systems Science and Engineering (JHU CSSE).

Both Microsoft Excel (spreadsheets) and Matlab (programming) were used for data processing and analysis.

\section{DISCUSSION}

\section{Understanding Death as a Rate}

First, a few simple definitions:

$C_{t}=$ number of confirmed Cases at time $=t$

$D_{t}=$ number of confirmed Deaths at time $=t$

$R_{t}=$ number of confirmed Recovered at time $=t$

$U_{t}=$ number of Unresolved cases at time $=t$

The above are simply related thus:
Cite this article: Patel SD, Nath P. Mid-Epidemic Case Fatality Rate - The Case of COVID-19: Reducing the Impact of Wrong Risk Perception. Int J Med Public Health. 2020;10(3):122-5. 
$C_{t}=D_{t}+R_{t}+U_{t}$

It is important to point out that each person represented by $U_{t}$ will at some future time $t+\delta$ be categorized as either $D_{t+\delta}$ or $R_{t+\delta^{*}}$.

Post-epidemic (PE), we have the usual definition of the Case Fatality Rate (CFR) based on all closed cases that would have been resolved by then as either 'dead' or 'recovered'. At that time:

$U_{P E}=$ number of unresolved cases "post-epidemic" $=0$

And equation (5) becomes:

$C_{P E}=D_{P E}+R_{P E}+U_{P E}$

And so (6) and (7) give us

$C_{P E}=D_{P E}+R_{P E}+0$

$C_{P E}=D_{P E}+R_{P E}$

We also have the classical $C F R_{P E}=$ Case Fatality Rate ("post-epidemic)"

$$
C F R_{P E}=\frac{D_{P E}}{C_{P E}}=\frac{D_{P E}}{D_{P E}+R_{P E}}
$$

This is a single point statistic to describe the end-point of the trajectory of the epidemic.

This is an appropriate moment to point out that the following cannot be used a logical measure of "death rate" or "risk of death".

$F-C F R_{t}=\frac{D_{t}}{C_{t}}=\frac{D_{t}}{D_{t}+R_{t}+U_{t}}$

We use the prefix " $F$ " to indicate that it is false. One must not include the number of unresolved cases in the denominator.

\section{What did the WORLD HEALTH ORGANIZATION CONVEY INCORRECTLY TO the Media?}

In the $3^{\text {rd }}$ March 2020 WHO Director-General's opening remarks at the media briefing on COVID19 the following statement was made. ${ }^{2 “}$ Globally, about $3.4 \%$ of reported COVID-19 cases have died. By comparison, seasonal flu generally kills far fewer than $1 \%$ of those infected." In those two sentences, the numerators, "number of deaths" are implicit but clear. Unfortunately, the globally media reporting to the public on the matter did not pick up the nuanced important difference between the denominators.

For the first sentence, the reference for COVID-19 is to reported cases, a significant proportion of which remained unresolved as at that date. In the second sentence, the reference for the season flu is to closed cases as it refers to the usual classical post-epidemic measure of CFR. Unfortunately, by using the phrase "by comparison', the media were inadvertently misled to believe that one could indeed make a "fair comparison". Here is why that impression is inappropriate using actual numbers as at that date.

The " $3.4 \%$ of reported" was obtained from:

$$
W \mathrm{HO}_{3 \text { rd March } 2020}=\frac{D_{3 r d M a r c h} 2020}{C_{3 r d M a r c h} 2020}
$$

$\mathrm{WHO}_{3 \mathrm{rd} \text { March } 2020}$

$$
=\frac{D_{3 \text { rd March } 2020}}{D_{3 \text { rd March } 2020}+R_{3 \text { rd March } 2020}+U_{3 \text { rd March } 2020}}
$$

As stated in equation (11) it is inappropriate to use this relationship (13) to represent any form of a fatality rate.

If it was the case that these unresolved cases as at $3^{\text {rd }}$ March 2020 were a tiny fraction of the big picture it would not matter. However, of the 92,840 confirmed cases as at that date, a whopping 41,452 i.e. $44.6 \%$ of all confirmed cases were unresolved.
$W \mathrm{HO}_{3 \mathrm{rd} \text { March } 2020}=\frac{3,160}{3,160+48,228+41,452}=3.4 \%$

In our opinion, even though they mention "reported cases", it was a gross error on the part of the WHO to have based their 3.4\% using all reported cases as it presented a scientifically inappropriate method.

What the media seemed to have understood and then conveyed to various sections of their followers was effectively the classical CFR:

Media $_{3 \text { rd March } 2020}=\frac{D_{3 \text { rd March } 2020}}{D_{3 \text { rd March } 2020}+R_{3 \text { rd March } 2020}}$

and as a result, the was $U_{3 r d \text { March } 2020}$ left hanging, set implicitly in their minds to be zero!

Whether that difference turns out to be material or not is a separate and important matter. What we have highlighted is the need to have a better method of representing mid-epidemic information. ${ }^{3}$ This is important for conveying messages to the public, including to the politicians who drive policies regarding public life.

\section{The Mid-Epidemic Case Fatality Rate (ME-CFR)}

We now formally introduce the equivalent concept when one is midepidemic. The Mid-Epidemic Case Fatality Rate (ME-CFR) is a measure of the fatality rate at all points in time when in the midst of an epidemic. More specifically, using the subscript $t$ for time again, we have

$M E-C F R_{t}=$ the case fatality rate at the specific point in time, $t$

With the typical variables from expressions (1), (2) and (3) as the only ones typically available and (4) being the balancing number, a suitable representation we can then have for the $\mathrm{ME}-\mathrm{CFR}_{\mathrm{t}}$ is:

$$
M E-C F R_{t}=\frac{D_{t}}{D_{t}+R_{t}+\beta_{t} U_{t}}
$$

In our representation in (16) we have introduced the time-varying parameter $\beta_{t}$ that we like to think of as being a blend parameter.

Central to much of what is in this paper is the phenomenon of the leadlag relationship between the variables that we measure and monitor. It is this relationship, along with the existence and mixing of multiple cohorts of infected individuals that introduces 'noise' into the statistics that are computed.

Let us assume, without loss of generality, that a typical infection takes $H$

\begin{tabular}{|c|c|c|}
\hline & $\begin{array}{l}\text { Until all the cases in the cohort are resolved } H \text { days later, we } \\
\text { cannot know the fraction of unresolved cases that are going to be } \\
\text { dead or recovered. }\end{array}$ & (17) \\
\hline • & $\begin{array}{l}\text { There is a lead-lag relationship between } D_{t} \text { on days } t=i,(i+1) \text {, } \\
(i+2) \ldots(i+H-1) \text { and } R_{i+H} \text { on day } t=i+H .\end{array}$ & (18) \\
\hline & $\begin{array}{l}\text { The only day on which it is correct to present the ratio } \frac{\boldsymbol{D}_{\boldsymbol{t}}}{\boldsymbol{C}_{\boldsymbol{t}}} \text { is } \\
\text { when } t=H \text {. }\end{array}$ & (19) \\
\hline & $\begin{array}{l}\text { Prior to } t=H \text { the ratio } \frac{D_{t}}{C_{t}} \text { will be biased downwards as not all } \\
\text { deaths for that cohort will be known to have occurred by then. }\end{array}$ & $(20)$ \\
\hline
\end{tabular}
days to be classified as 'recovered'. For a given single cohort of individuals $C_{i}$ infected on day $t=i$, we can have one or more deaths on any day between day $t=i$ and day $t=i+H$. This implies a number of facts:

In reality when we have multiple such cohorts of varying sizes, varying recovery periods and varying time-to-death, the invalidity of equation (13) to produce an informative number does not go away.

Having appreciated that when we are mid-epidemic, the elephant in the room is Ut the unresolved cases that are going to at some point end up being classified as either 'dead' or 'recovered' the time-varying parameter $\beta_{t}$ helps us understand the animal we wish to tame.

$\beta_{t}=$ the proportion of $U_{t}$ that will recover in the future, after time $=t$ 
Setting $\beta_{t}=1$ is clearly wrong if not everyone whose case is yet unresolved will recover. Setting $\beta_{t}=0$ is clearly wrong if not everyone whose case is yet unresolved will die.

Expression (21) tells us that it is useful to think of as relating to the future despite it having the subscript $t$. As a result, even though the subscripts $t$ for $C_{t}, D_{t}, R_{t}$ and $U_{t}$ relate to a measurement reported at time $t$, for past events up to time $t$, we can subsume the future path of fatalities into and focus on controlling its trajectory. Figure 1 illustrates this.

Within a population, $\beta_{t}$ reflects the combined ability of the individual, through their immune system response and the healthcare infrastructure, to transition that individual from a state of infection to a state of full recovery. This can be described further using the COVID-19 pandemic as an example.

If we imagine the case that a perfect pharmacological cure is found and can be administered on all those infected, $\beta_{t}$ will move towards 1 .

On the other hand, in a healthcare system where patient support has turned abysmal because, for instance, there is a shortage of ventilators, the $\beta_{t}$ will move towards 0 .

As the ME-CFR is for 'mid-epidemic' it is up to the user to decide when one is in the middle of an epidemic. For a pandemic, it may well be that for a given country unaffected, or with only a handful of infections, there is no real epidemic. For example, as of $26^{\text {th }}$ April, a country like the Seychelles that gets its fair share of tourists throughout the year, there were only 11 recorded cases (and no deaths). At the other end of time, post-epidemic, we are aware that the classical CFR and the MECFR will coincide numerically as the $\mathrm{U}_{\mathrm{PE}}$ will be zero. Our focus is on

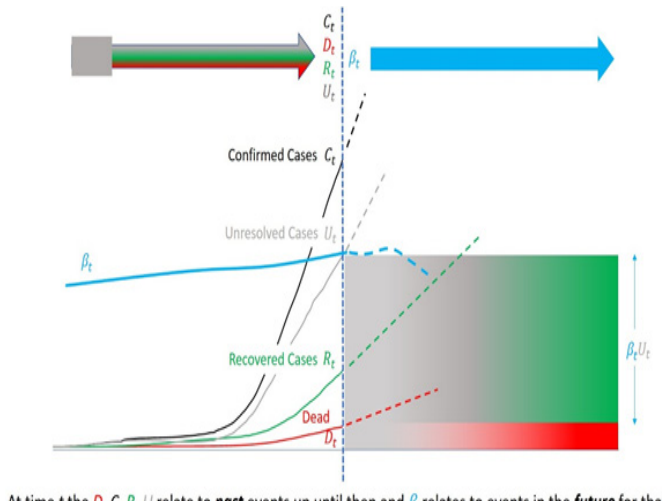

Figure 1: At time $t$ the $D, C, R, U$ relate to past events up until then and $\beta$ relates to events in the future for that $\mathrm{U}$.

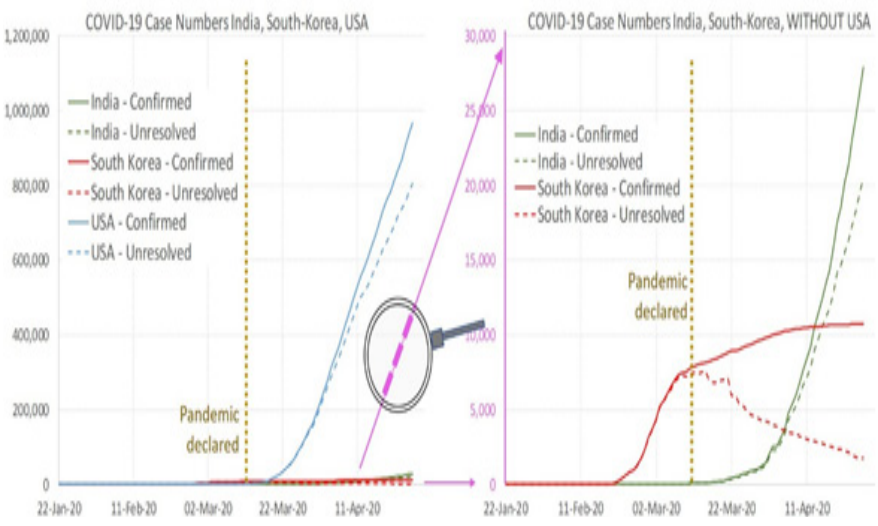

Figure 2: COVID-19 Case Numbers for South Korea, India, USA. mid-epidemic and for cases that are confirmed infections, i.e. we do not address the joint event of transmission potential and virulence. ${ }^{4}$

\section{ME-CFR for the COVID-19 Pandemic}

It was the $11^{\text {th }}$ March 2020 declaration by the WHO that started the era of the COVID-19 pandemic as being a pandemic.

We look at only the following three countries: India, South Korea and the USA.

One can engage in a long debate as to why only three countries and why those three. Our preference was to cover reliability of numbers, scale of population, GDP, wealth per capita, policies imposed and compliance to commonly acceptable social norms. Unless specified, we present the countries in alphabetical order.

The data is freely available from the Johns Hopkins COVID-19 data repository on Git Hub.

Before we dive into the ME-CFR which is a relative number, we set the stage by presenting the absolute numbers. The data commences on 22-January-20 and the last date for which data is presented here is 26 Apr-20.

As can be seen from Figure 2, by the end of the data period, the absolute numbers for the USA are almost an order of magnitude larger than for India and South Korea. Therefore, rather than present numbers on a logscale, which is not easy for humans to visualize, we present alongside the numbers for India and South Korea, excluding the USA.

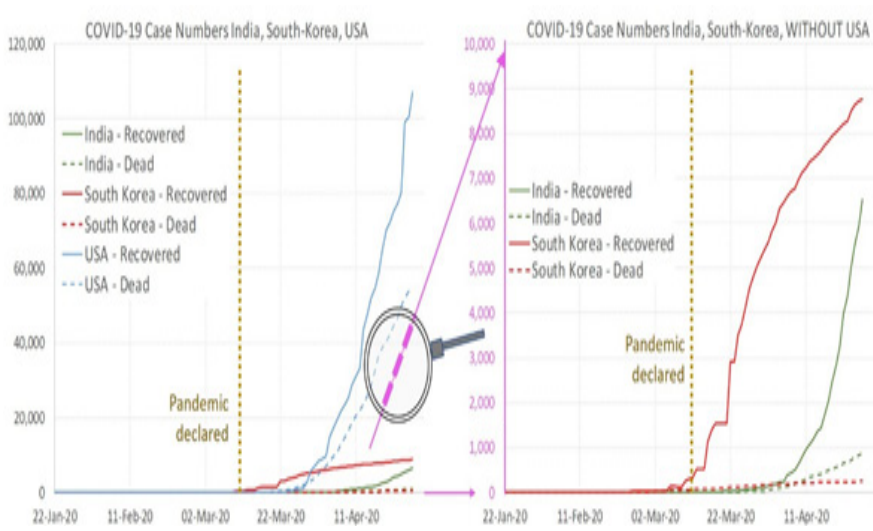

Figure 3: COVID-19 Dead and Recovered Numbers for South Korea, India, USA.

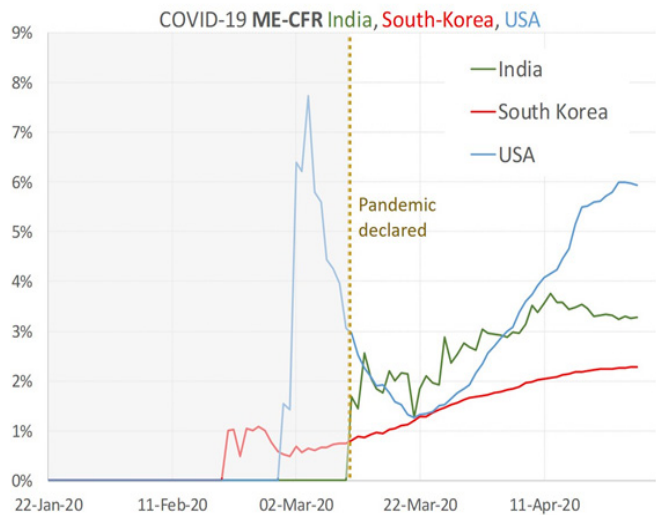

Figure 4: Mid-Epidemic CFR for South Korea, India, USA. 
Unfortunately, for the USA, the number of deaths is at least an order of magnitude higher than for India and South Korea as can be seen in Figure 3.

Now, we present the ME-CFR, Mid-Epidemic Case Fatality Rate, based on our framework using a single $\beta_{t}=0.95$. Figure 4 suggests many stories. India, with its very large population and high population density, reduced its downside risk of a very large absolute number of deaths with a nationwide lockdown from $21^{\text {st }}$ March 2020 until and beyond the date of writing this paper. This has allowed its healthcare infrastructure to keep working appropriately to keep the ME-CFR very much under control.

South Korea, with large numbers of tests per capita and effective use of technology for contract tracing, has kept its ME-CFR low thanks also to very good compliance in social distancing recommendations.

The USA has faced a lot of internal conflict and criticism within its own borders about how it has gone about handling the COVID-19 pandemic. Unfortunately, at the time of writing this, it seems like the ME-CFR for the USA is likely to climb higher before it too, hopefully, settles down at a lower level.

\section{CONCLUSION}

Whereas thinking of a case fatality rate makes us feel like passive recipients of what nature sent our way in the past, the time-varying parameter $\beta_{t}$ of the ME-CFR empowers us by reminding us that we can control the future with our choices and actions. This subtle difference is powerful because appropriate beliefs will engender social behaviour within a healthcare system that will be more effective in also limiting the rate at which an infection spreads within the wider population.

\section{CONFLICT OF INTEREST}

The authors declare no conflicts of interest.

\section{ABBREVIATIONS}

CFR: Case Fatality Rate; COVID-19: Coronavirus disease 2019; MECFR: Mid Epidemic Case Fatality Rate.

\section{REFERENCES}

1. Allan MB, Alyssa B. Not a Perfect Storm - Covid-19 and the Importance of Language. N Engl J Med. 2020;382:1493-5.

2. World Health Organization. Geneva: WHO Director-General's opening remarks at the media briefing on COVID-19 - 3 March 2020. 2020. [cited 2020 Mar 03] Available from: https://www.who.int/dg/speeches/detail/who-director-generals-opening-remarks-at-the-media-briefing-on-covid-19--3-march-2020

3. David B, Xiaolong Q, Nielsen-Saines K, Didier M, Léo P. Guillaume Favre Real estimates of mortality following COVID-19 infection. 2020. DOI:https://doi. org/10.1016/S1473-3099(20)30195-X

4. Keisuke E, Ryosuke O, Benjamin JC, Kazuyuki A, Hiroshi N. The Time Required Estimating the Case Fatality Ratio of Influenza Using Only the Tip of an Iceberg: Joint Estimation of the Virulence and the Transmission Potential. Computational and Mathematical Methods in Medicine. 2012. [cited 2012 May]; Article ID 978901, doi:10.1155/2012/978901: [about 10 pages]Available from: https:// www.hindawi.com/journals/cmmm/2012/978901/

Cite this article: Patel SD, Nath P.. Mid-Epidemic Case Fatality Rate - The Case of COVID-19: Reducing the Impact of Wrong Risk Perception. Int J Med Public Health. 2020;10(3):122-5. 